\title{
Biomimetic Bonelike Composites and Novel Bioactive Glass Coatings
}

\author{
A. P. Tomsia, ${ }^{\#}$ E. Saiz, ${ }^{\#}$ J. Song, ${ }^{\#}$ C. Bertozzi ${ }^{\#, \dagger}$ \\ "Materials Sciences Division \\ Lawrence Berkeley National Laboratory \\ Berkeley, CA 94720, USA \\ ${ }^{\dagger}$ Departments of Chemistry, Molecular and Cell Biology and Howard Hughes Medical \\ Institute, University of California, Berkeley, California 94720, U.S.A.
}

\begin{abstract}
Metallic orthopaedic implants have been successfully used for decades but they have serious shortcomings related to their osseointegration and the fact that their mechanical properties do not match those of bone. This paper reviews recent advances in the fabrication of novel coatings to improve implant osseointegration and in the development of a new generation of hybrid organic-inorganic implant materials specifically designed for orthopaedic applications
\end{abstract}

\section{Introduction}

The number of orthopedic surgeries performed worldwide is growing 10 to $12 \%$ annually. ${ }^{[1]}$ As these procedures become more popular, and patients desire to lead more active lives, these rates are expected to increase. An estimated 11 million people in the U.S. have at least one medical-device implant and more than $\$ 50$ billion are spent annually on reconstructive devices. ${ }^{[2]}$ In 2003 alone, more than 700,000 dental implant procedures were performed in the U.S., and more than 1.3 million in Europe; ${ }^{[3]}$ sales in 2003 for implants and devices were $\$ 8.7$ billion and are expected to rise at an annual growth rate of $12.5 \%$ and reach $\$ 17.9$ billion by $2009 .^{[1]}$ Current commercial implants are fabricated using materials typically developed for other engineering applications, and their osseointegration and long-term stability are still a source of concern. The National Center for Health Statistics reports that nearly $15 \%$ of the hip replacement surgeries performed in 2002 were to revise original replacements, ${ }^{[4]}$ and this number is likely to increase as younger people undergo these procedures. These numbers emphasize the need for a coordinated research effort to provide patients and doctors with better and more durable implants. A short- to medium-term solution is the modification of implant surfaces to enhance bonding to bone. In the long term, new implant materials-materials that will match the mechanical properties of bone and be actively integrated and remodeled by the body_-should be developed. This paper reviews some of the recent efforts in the development of bioactive glass coatings to enhance the osseointegration of metallic and ceramic implants, and the most recent research in the field of organicinorganic hybrid composites specifically designed for bone replacement. 


\section{Novel Coatings}

Metals have been used successfully for decades in fracture fixation, joint replacement, and dental applications. ${ }^{[5]}$ More recently, high-strength technical ceramics are also being considered for the fabrication of orthopedic implants. ${ }^{[6]}$ In many cases, surface treatments are used to improve the materials' bonding ability to living tissues, particularly bone. Different approaches are under investigation, including texturing or chemical modification of the surfaces to enhance cell attachment, large-scale roughening to promote bone ingrowth with the result of an improved mechanical bonding, or applying calcium phosphate coatings by different methods to create osteophilic surfaces that will bond chemically to the bone. ${ }^{[7]}$ The most popular coating procedure is plasma spray.$^{[7]}$ Plasma-sprayed coatings are now commercially available, and numerous studies have shown that they enhance interfacial attachment to bone tissue over short periods of time, and promote the formation of a more reliable interface with bone than mechanical osseointegration. ${ }^{[7]}$ However, the calcium phosphate coatings fabricated by plasma spray have severe drawbacks that include: (1) cracking and unreliable adhesion; (2) variability during processing in terms of resulting phases (typically, the coatings consist of a mixture of phases, some of them highly soluble amorphous calcium phosphates), morphology, thickness, and stresses; (3) significant degradation in the fatigue resistance and endurance strength of the implant alloy, and (4) inability to systematically change the coating attributes. $^{[5,7]}$

Clearly, bioactive coatings can improve implant performance, but we are still far from the development of optimum materials and fabrication technologies. Ideally, the coatings should be tailored to exhibit prescribed biological attributes for each specific application. They should have strong adhesion to the implant and good fixation to bone. Their composition and microstructure should be controlled such that their dissolution rates match the in vivo healing process. The coatings could also serve as templates for the in situ delivery of drugs and growth factors at the required times. Most likely this will not be achieved by using a single procedure or material, but by combining several of them to fabricate layers that blend organic and inorganic phases, with thicknesses ranging from hundreds of microns to the nanometer level, and chemically and topographically textured surfaces.

The potential of bioactive glass coatings was recognized since the discovery of the original Bioglass ${ }^{\circledR}$ composition by Hench. ${ }^{[8]}$ Bioglass ${ }^{\circledR}$ has excellent bioactivity and could be used to enhance the adhesion of the implant to the bone. The development of bioactive glass coatings could take advantage of the extensive accumulated experience on glass-metal bonding and sealing. Furthermore, the glass properties can be easily adjusted from bioactive to bioresorbable or bioinert by controlling their composition. ${ }^{[8]}$ This creates the possibility of fabricating layers with finely tuned properties. However, initial attempts to coat metallic implants with the original Bioglass ${ }^{\circledR}$ were marred by the generation of large thermal expansion stresses and high reactivity between the metal and the glass. ${ }^{[9]}$ Subsequently, several techniques have been investigated for the preparation of glass coatings, such as enameling, plasma spray, RF sputtering, sol-gel, pulsed-laser deposition (PLD), and others. Although it has been possible to deposit glass layers that retain bioactivity, a recurrent problem is the control of the glass-metal reactions to 
achieve good coating adhesion, and the generation of large thermal stresses during processing that can cause cracking and/or delamination of the coating. The solution to the problem requires the development of coating procedures and novel bioactive glasses, and glass-ceramics, with adequate thermal expansion coefficients and softening points.
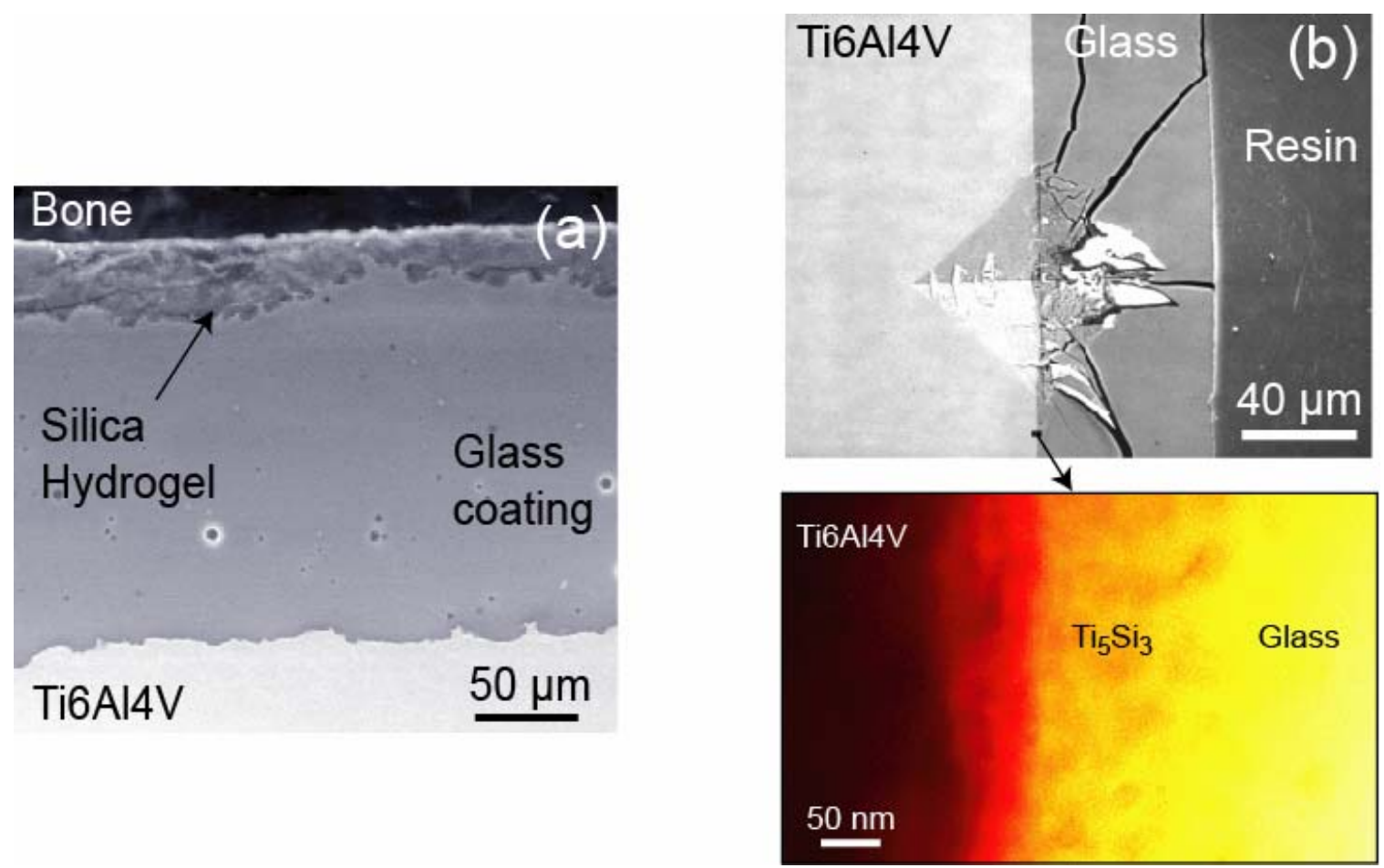

Figure 1. (a) Environmental SEM showing a cross section of a graded glass coating after a 6-month in vivo test in a pig model. The coating survived, maintaining excellent adhesion to the implant. A corroded layer can be observed on the coating surface providing adhesion to the bone, but the high-silica layer in contact with the alloy remains intact, maintaining good adhesion to the metal. Preliminary tests indicate that, after 6 months, the bone matrix in the vicinity of the bone-implant interface of coated implants had changed to lamellar-type bone, while the uncoated implants remained with woventype bone. It is reasonable to assume that the coated device had less micromovement, so that the biomechanical integration of the device to the surrounding bone tissues was achieved earlier during the experimental period, facilitating the transformation of wovenbone matrix to lamellar type over a 2- to 6-month time period. (b) During indentation tests, the cracks do not propagate at the glass/metal interface, indicating good coating adhesion achieved through the formation of a thin (100-150 nm) $\mathrm{Ti}_{5} \mathrm{Si}_{3}$ layer that can be observed using high-resolution TEM.

Since the discovery of Bioglass ${ }^{\circledR}$, other bioactive glass compositions have been reported in the literature. In our laboratory, we have demonstrated that a new family of bioactive glasses, in the system Si-Ca-Mg-Na-K-P-O, presents thermal expansion coefficients close to those of the metallic alloys used in orthopedic implants. ${ }^{[10]}$ This is achieved through the partial substitution of $\mathrm{Ca}$ by $\mathrm{Mg}$, and of $\mathrm{Na}$ by $\mathrm{K}$. These glasses have adequate softening points to be used in the fabrication of coatings by a simple enameling 
technique. Strong glass/metal adhesion is achieved through the formation of thin interfacial layers. The procedure can be extended to fabricated graded coatings through the sequential deposition of different glass and glass-ceramic layers. For example, we have fabricated graded coatings on $\mathrm{Ti}$ and $\mathrm{Co}-\mathrm{Cr}$ alloys consisting of a high-silica glass in contact with the metal, and a composite surface layer that consists of a mixture of a lowsilica glass with synthetic hydroxyapatite (HA) particles (Figure 1). The high-silica layer is very resistant to corrosion in body fluids; it provides good coating adhesion and longterm stability, while the surface layer is designed to enhance coating attachment to the tissue (Figure 1).
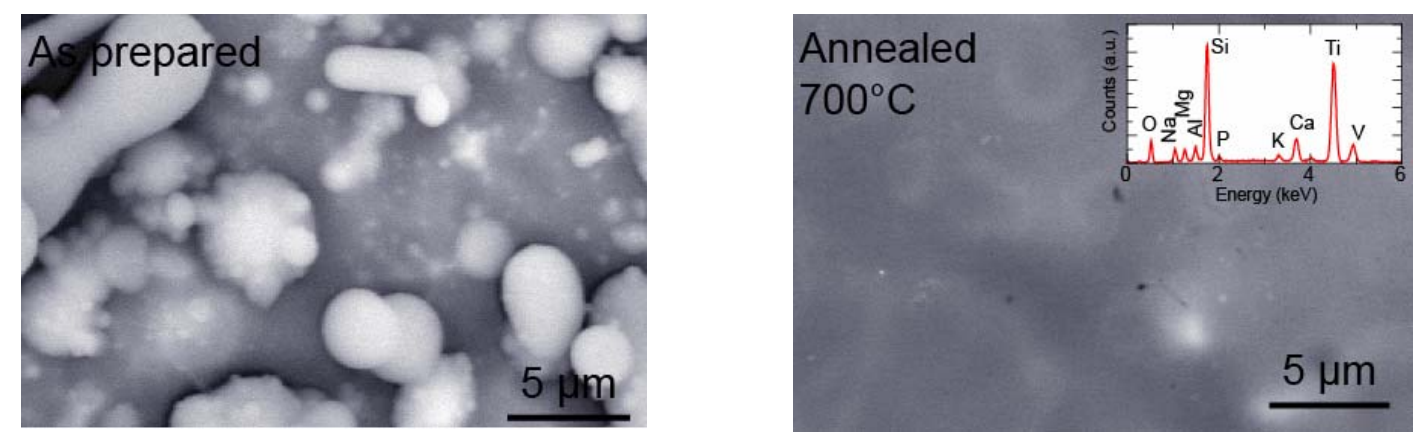

Figure 2. Surface of a thin glass coating deposited on Ti6AI4V by PLD, as prepared and after annealing for $30 \mathrm{~s}$ at $700{ }^{\circ} \mathrm{C}$. The coating is thin and the substrate peaks are visible in the EDS analysis. Quantitative analysis indicates that the stoichiometry of the layer is in $\pm 5 \mathrm{wt} \%$ of the original glass target.

Techniques such as enameling or plasma spray can be used to fabricate relatively thick layers, typically 50 to $200 \mu \mathrm{m}$. However, the driving force for coating cracking and delamination increases with coating thickness; thinner layers could withstand much larger thermal stresses and have a wider range of compositions. Perhaps two of the most researched techniques for the fabrication of thin bioactive glass layers are sol-gel and pulsed-laser deposition. ${ }^{[11,12]}$ Both techniques can be adapted to the fabrication of multilayered coatings. However, the fabrication of glass layers with complex compositions by sol-gel could be problematic. On the other hand, PLD can be used to prepare thin films of any material, including complex ceramics and glasses, while maintaining coating stoichiometry (Figure 2). In PLD, a high-power laser is focused onto a target. The extreme energy density at the focus breaks many chemical bonds, and a few surface layers are ejected at high kinetic energy in the form of an ablation "plume." The components of these layers travel ballistically across the vacuum chamber until they strike a substrate material. The high-impact energies (typically $100 \mathrm{eV}$ ) cause the particles to stick to the surface and be compressed, forming a dense continuous film. PLD can be used to deposit highly bioactive and hygroscopic glasses, or glasses with very high softening points that cannot be applied by other methods. By controlling the target temperature, or annealing after deposition, coating adhesion and surface roughness can be manipulated. It has been observed that the implant surface texture affects cell attachment and osseointegration. Rapid prototyping techniques such as ink-jet printing or robocasting can be used to fabricate textured glass coatings (Figure 3). These techniques 
can also be used to integrate organic materials into coatings to serve as templates for the delivery of drugs and growth factors.

Glass-based coatings offer a unique versatility; they can be applied using a wide range of techniques, and their composition — and therefore their properties — can be easily manipulated. It is well known that bioactive glasses can generate hydroxyapatite (a calcium phosphate closely related to the mineral component of the bone) in vivo though a complex dissolution-precipitation process. The interaction of glass coatings with tissue can be further manipulated by designing appropriate surface textures. Furthermore, in vitro analysis in cell cultures has shown that bioactive glasses and coatings can indirectly affect osteoblast gene expression through their dissolution products. Quantitative realtime RT-PCR (reverse transcription-polymerase chain reaction) analysis showed that silicate glass coating extract induced a twofold expression of Runx-2, a key marker of osteoblast differentiation, compared to Ti-6Al-4V and tissue culture polystyrenes. Future investigation should address the identification of specific chemical cues behind the induction of gene expression, and the tailoring of layer compositions to control such effects.

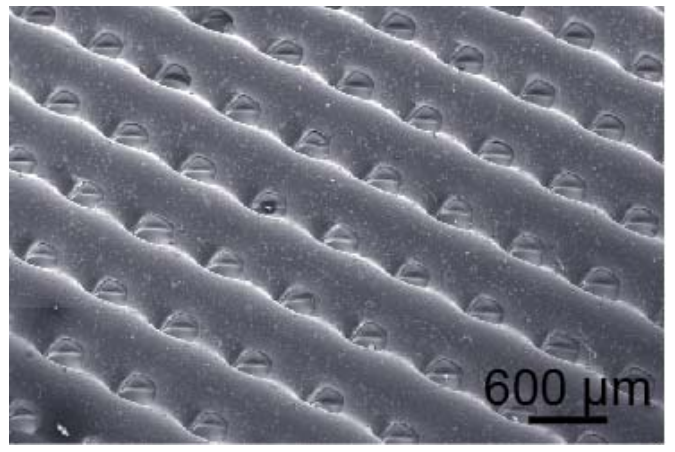

Figure 3. Textured glass coating surface printed on Ti6Al4V using robotic assisted deposition. The glass belongs to the Si-Ca$\mathrm{Mg}-\mathrm{Na}-\mathrm{K}-\mathrm{P}-\mathrm{O}$ and has a silica content of $53 \mathrm{wt} \%$. The ink has a 70 wt $\%$ content of glass dispersed in ethanol, with $0.8 \mathrm{wt} \%$ of a dispersant (Mellorian 312, CECA S.A.), $2.5 \mathrm{wt} \%$ Methocell F4M (7.3 wt\% in water), and $0.5 \mathrm{wt} \%$ polyethilenimine.

\section{Bonelike Composites}

Even if the use of coatings can improve their osseointegration, current implants still have a fundamental flaw: they are foreign objects stiffer than bone. Consequently, the implants bear a greater proportion of the load than the surrounding skeleton, shielding it from its normal stress level. The osteoblasts are not stimulated into generating new bone, the tissue is resorbed and the implant becomes loose over time, limiting its life. Thus, an ideal solution, and a scientific challenge, is to develop bone-like biomaterials that will be treated by the host as normal tissue matrices, and induce cell penetration and proliferation after implantation, while their properties match those of the tissue to be repaired. These adaptive biomaterials will integrate with bone tissue while they are actively resorbed or remodeled in a programmed way, with controlled osteogenic activity. Such implants could also act as drug delivery systems by releasing drugs and/or stimulating the growth of new bone tissue at the fracture site by slowly releasing bone growth factors (e.g., bone morphogenic protein or transforming growth factor- $\beta$ ) throughout its degradation process. This is an optimal solution if the requirements of strength and performance can 
be met, since natural tissues can replace and repair themselves. To accomplish this, the strength and stability of the material-tissue interface should be maintained while the material is resorbed or remodeled, and its resorption and remodeling rates should be matched to the tissue repair rates, which can vary enormously with tissue type or patient age. It is also important that biodegradation of the resorbable material results only in production of metabolically acceptable substances. The materials will then take advantage of the biological principles of bone repair that have been developed over millions of years of evolution.
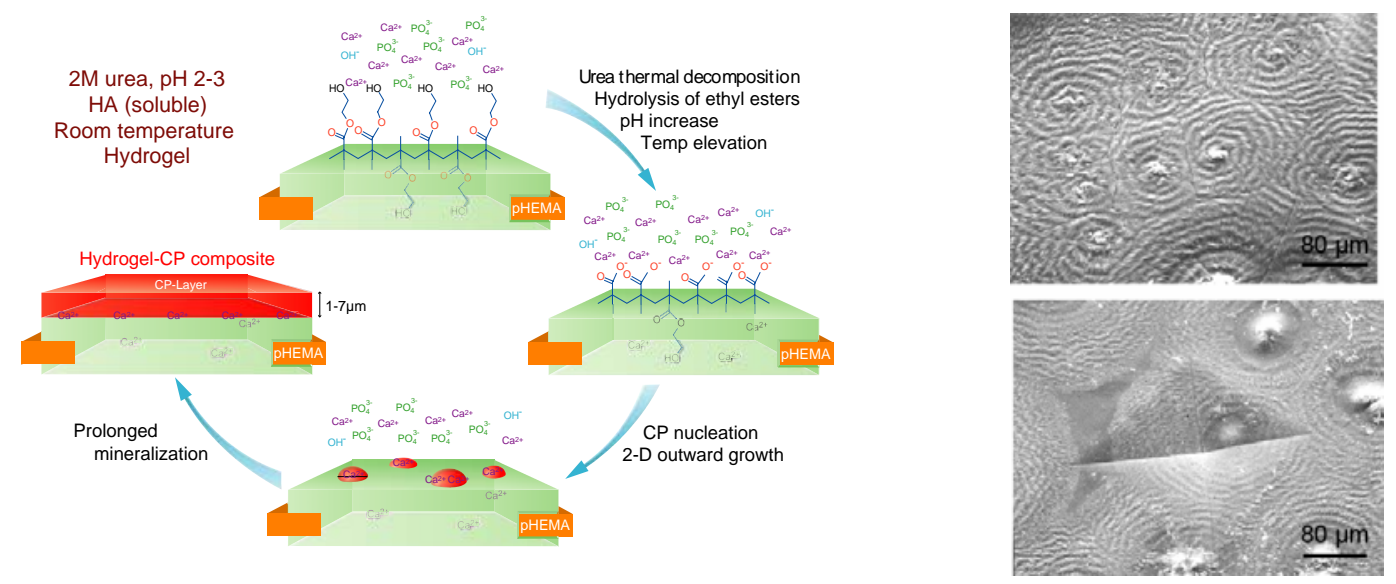

Figure 4. Urea-mediated solution mineralization of hydroxyapatite (HA) onto pHEMA hydrogel scaffolds. Thermo-decomposition of urea produces a gradual increase in $\mathrm{pH}$, resulting in the hydrolysis of surface 2-hydroxyethyl esters and the precipitation of HA from the aqueous solution. The in situ generated surface carboxylates strongly interact with calcium ions and facilitate the heterogeneous nucleation and 2-dimensional growth of a high-affinity calcium-phosphate (CP) layer on the pHEMA surface. Prolonged mineralization allows for the growth of a thicker CP layer that covers the entire hydrogel surface. The SEM microcraph on the right shows the 2-dimensional circular outward growth of a calcium apatite layer from multiple nucleation sites on the acidic surface of pHEMA. The calcium phosphate layer did not delaminate, even after Vickers indentation with a load of $5 \mathrm{~N}$ (bottom micrograph).

The challenge can be matched with the use of organic-inorganic composites that will combine the toughness of the organic phase with the stiffness of the inorganic material. The adequate combination of biodegradable polymers and ceramics can be used to manipulate the resorption of the composite. However; fabrication of those materials has proved difficult. Several groups have reported the fabrication of organic-inorganic composites by mixing biodegradable polymers such as poli-L-lactide with micron-sized hydroxyapatite particles. ${ }^{[13]}$ Whereas some of these materials have interesting properties, very often the composites are marred by low elastic modulus, poor mechanical properties, or fast degradation in the biological environment. ${ }^{[14]}$ It is necessary to develop novel processing routes where the material microstructure can be controlled from the nano to 
the meso level. In this way, the structure of natural bone can be used as a blueprint for the design of new materials.

Structurally, natural bone is a composite of collagen, a protein-based hydrogel template, and inorganic dahilite (carbonated apatite) crystals. The microstructural design of bone, combined with the controlled integration of the organic and inorganic components, provides unique properties: low density and stiffness, and relatively high strength and toughness. Throughout the cavities of bone, there are bone cells and a myriad of soluble factors and extracellular matrix components that are constantly involved in the bone remodeling process. Bone biogenesis is thought to occur by templated mineralization of hard apatite crystals by the elastic collagen scaffold, a process that several research groups have sought to emulate by the synthetic biomimetic mineralization of different organic materials. For example, hydroxyapatite crystals have been grown on collagen scaffolds using mineralizing solutions. However, reports of the final properties of these materials are scarce. ${ }^{[15]}$ Most of them seem to lack the level of organic-inorganic integration observed in real bone. On the other hand, Hartgerink et al. ${ }^{[16]}$ have demonstrated that peptide-amphilides can self-assemble to form fibrous scaffolds that will template the mineralization of HA in a way that mimics the alignment between collagen fibrils and apatite crystals in bone.

A particularly appealing candidate for the design of highly functional organic scaffolds for mineralization is hydrogel polymers. The intrinsic elasticity and water retention ability of synthetic hydrogels resemble those of collagen, and their porosity may be controlled by various techniques. Another important feature of hydrogels is that they can be assembled in 3-dimensional form, displaying multiple functional domains through copolymerization of different monomers. The polymerization chemistry is water compatible, allowing incorporation of polar ligands such as anionic peptides that mimic the acidic matrix proteins regulating mineral growth, and biological epitopes such as the tripeptide RGD that promote cellular adhesion. Observations have shown that pHEMAbased hydrogel copolymers can be mineralized in a way that provides very strong polymer-mineral interfacial adhesion. ${ }^{[17,18]}$ The mineralization method takes advantage of the dramatically different solubilities of HA in acidic and basic aqueous solutions, and the chemically labile nature of ester groups of pHEMA in basic solutions. Thermodecomposition of urea in aqueous media was used as a facile $\mathrm{pH}$ modulator to generate an anionic surface and partially acidic interior of the pHEMA gel (Figure 4). This initiated the heterogeneous nucleation and high-affinity growth of calcium apatite on the gel surface, and extensive calcification inside the gel. The intrinsic chemical nature of the hydrogel surface (the in situ generation of surface carboxylates that strongly bind to calcium ions) dictates the high-affinity template-driven mineralization observed with a strong hydrogel-mineral adhesion (Figure 4). In addition, cross-linked polymethacrylamide and polymethacrylate hydrogels can be functionalized with mineralbinding ligands and used to template the formation of hydroxyapatite. Strong adhesion between the organic and inorganic materials can be achieved for hydrogels functionalized with either carboxylate or hydroxy ligands. ${ }^{[17]}$ The mineral-nucleating potential of hydroxyl groups identified here broadens the design parameters for synthetic bonelike 
composites and suggests a potential role for hydroxylated collagen proteins in bone mineralization. Human osteoblastic cells were found to attach, spread, and proliferate on all synthetic hydrogel copolymers tested, with no apparent cytotoxicity.

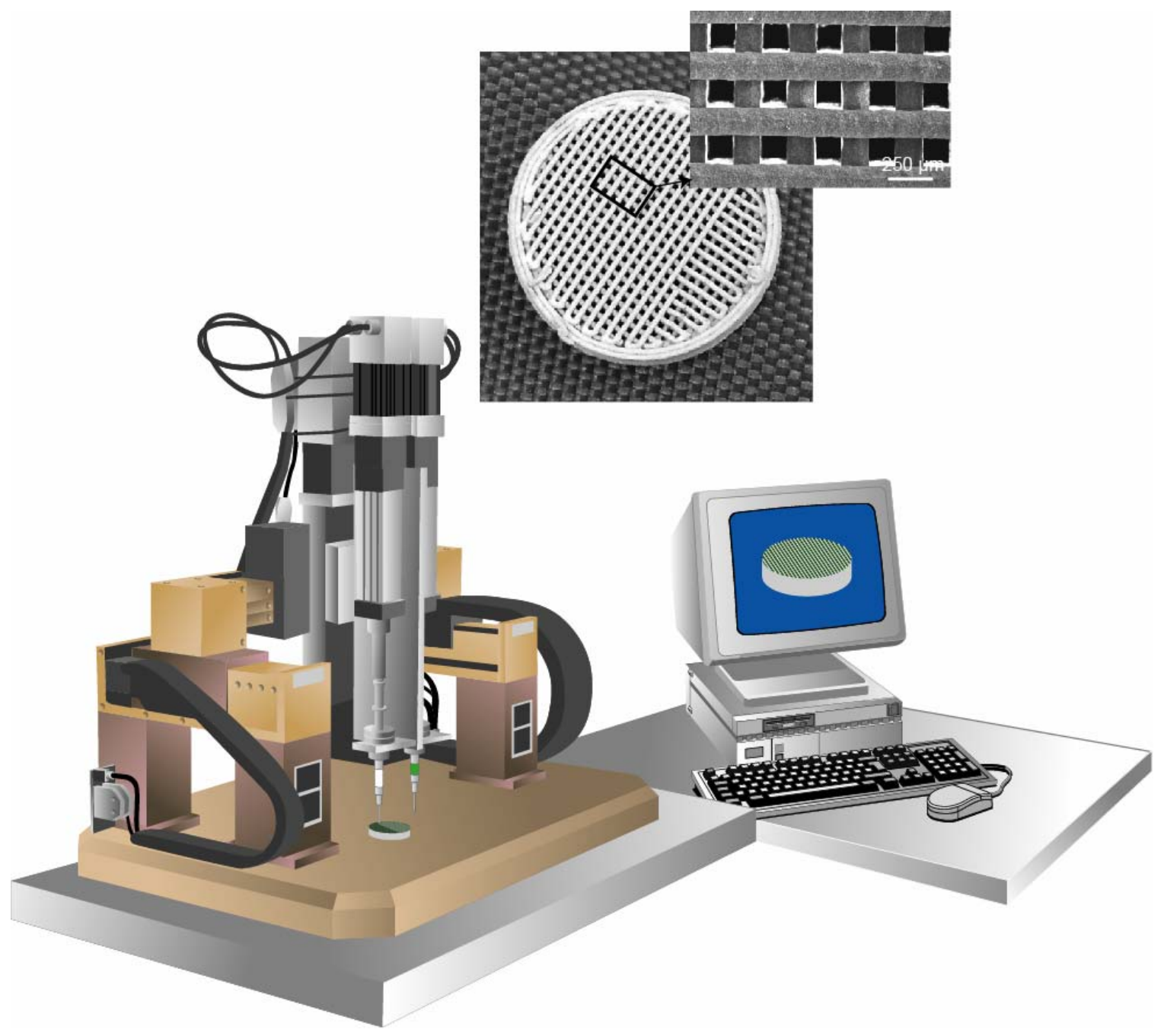

Figure 5. Schematic of the robocasting process a computer aided design technology for the free-form fabrication of ceramic scaffolds. A ceramic slurry is extruded through a thin nozzle to build a part layer by layer following a computer design. The slurry must exhibit a controlled viscoelastic response. It should flow through the nozzle and subsequently settle very fast, bonding to the previous layer so that the part maintains its shape while printing. The slurries should contain a high solid fraction (typically between 40 to 60 wt\%) to minimize drying-induced shrinkage after assembly. The insets show a HA grid fabricated by robocasting.

A reverse approach is to fabricate porous ceramic scaffolds, with controlled microstructure, as the base for the fabrication of dense organic-inorganic composites through infiltration with polymers. Techniques for the fabrication of porous inorganic 
materials are well developed, and with the advent of the so-called solid-free-form fabrication technologies, or rapid prototyping technologies, we can now custom design and produce porous scaffolds directly from a computer-aided design (CAD) model with determined shapes and porosities (Figure 5). ${ }^{[19]}$ These techniques produce 3-D objects guided by a CAD file, as well as digital data produced by an imaging source such as computed tomography (CT) or magnetic resonance imaging (MRI), opening the door for the fabrication of custom-designed implants. Gradients in material porosity, as well as sequential infiltration of polymers with different resorbability, can be used to further manipulate the material resorption rate.

Both strategies have their advantages. While biomineralization of organic scaffolds is a process closer to the formation of natural bone, starting from porous inorganic materials could be a shorter route to the fabrication of novel biomaterials customized for diverse applications. These materials can also be tailored for systematic in vitro and in vivo studies that will reveal the mechanisms of implant fixation and durability, and correlate them with the materials' behavior in cell cultures.

\section{Summary}

The initial search for strong orthopedic materials led to the use of bioinert alloys or ceramics. These implants have improved the lives of thousands of patients worldwide, and will probably be used for a long time. However, most implant limitations are still material related. Two important concerns are their osseointegration and their extreme stiffness. Glass coatings can be a viable alternative to current surface treatments used to improve implant adhesion to bone. The composition of the glasses can be adjusted in order to modulate coating properties. Different techniques can be used in order to fabricate coatings with a wide range of thickness, texture, and gradient. The glasses could be combined with other organic or inorganic materials to tune the coating resorption rates or to fabricate therapeutic coatings. However, it is necessary to develop new materials specifically designed for orthopedic applications. This challenge could be met by novel hybrid organic/inorganic composites with an architecture controlled from the nano to the mesolevels. The development of coatings and materials is intimately related, and the lessons learned in one field can be applied in the other. Fundamental research is necessary to develop novel compositions and fabrication techniques, and to better understand the microstructural parameters that control the materials' response in vitro and in vivo. A key component of the effort should be the analysis of structure and adhesion at both the internal material interfaces (glass-metal, polymer-ceramic) and the implant-tissue interface. This development can only take place through an interdisciplinary effort combining materials scientists, chemists, physicists, biologists, and clinicians. The end result will not only be better biomaterials, but a better understanding of the natural tissues, and lessons that can be applied to the fabrication of materials for many other applications. 


\section{Acknowledgements}

This work was supported by the National Institutes of Health (NIH) under Grants No. 5R01 DE015633 and 1R01DE11289.

\section{References}

[1] Shahani, S. “Orthopedic Drugs, Implants and Devices,” Business Communications Company Inc. Market Study, 2004.

[2] Praemer, A.; Furner, S.; Rice, D. P. Musculoskeletal Conditions in the United States, American Academy of Orthopaedic Surgeons.1992.

[3] Press release "Henry Schein Enters Growing Dental Implant Category Through Strategic Partnership with Camlog." In Bussines Wire, 2004.

[4] Number of Patients, Number of Procedures, Average Patient Age, Average Length of Stay - National Hospital Discharge Survey 1998-2002. In National Hospital Discharge Survey 1998-2002; Statistics., U.S. Department of Health and Human Services; Centers for Disease Control and Prevention; National Center for Health Statistics

[5] Davies, J. E. Bone Engineering; EM Squared: Toronto, 2000.

[6] Pecharroman, C.; Bartolome, J. F.; Requena, J.; Moya, J. S.; Deville, S.; Chevalier, J.; Fantozzi, G.; Torrecillas, R. Adv. Mater. 2003, 15, 507.

[7] Willmann, G. Adv. Eng. Mater. 1999, 1, 95.

[8] Hench, L. L. J. Amer. Ceram. Soc. 1991, 74, 1487.

[9] Andersson, O.; Hench, L. L., in An introduction to bioceramics; (Eds: L. L. Hench, J. Wilson), World Scientific: New Jersey 1993, 239.

[10] Lopez-Esteban, S.; Saiz, E.; Fujino, S.; Oku, T.; Suganuma, K.; Tomsia, A. P. J. Eur. Ceram. Soc. 2003, 23, 2921.

[11] Duran, A.; Conde, A.; Coedo, A. G.; Dorado, T.; Garcia, C.; Cere, S. J. Mater. Chem. 2004, 14, 2282.

[12] Serra, J.; Liste, S.; Gonzalez, P.; Serra, C.; Borrajo, J. P.; Chiussi, S.;

Leon, B.; Perez-Amor, M. Appl. Phys. A-Mater. Sci. \& Proc. 2004, 79, 983.

[13] Burg, K. J. L.; Porter, S.; Kellam, J. F. Biomaterials 2000, 21, 2347.

[14] Zhang, Y.; Wise, D. L.; Hsu, Y.-Y., in Biomaterials and Bioengineering Handbook; (Ed: D. L. Wise), Marcel Dekker: New York, 2000, 553.

[15] Lawson, A. C.; Czernuszka, J. T. Proceedings of the Institution of Mechanical Engineers Part H-Journal of Engineering in Medicine 1998, 212, 413.

[16] Hartgerink, J. D.; Beniash, E.; Stupp, S. I. Science 2001, 294, 1684.

[17] Song, J.; Malathong, V.; Bertozzi, C. R. J. Amer. Chem. Soc. 2005, 127, 3366.

[18] Song, J.; Saiz, E.; Bertozzi, C. R. J. Amer. Chem. Soc. 2003, 125, 1236.

[19] Tay, B. Y.; Evans, J. R. G.; Edirisinghe, M. J. Inter. Mat. Rev. 2003, 48, 341. 